\title{
Proton Pump Inhibitor-Related Gastric Mucosal Changes
}

\author{
Gwang Ha Kim \\ Department of Internal Medicine, Pusan National University College of Medicine and Biomedical Research Institute, Pusan National \\ University Hospital, Busan, Korea
}

\section{Article Info}

Received January 31, 2020

Revised March 25, 2020

Accepted March 30, 2020

Published online April 27, 2020

\section{Corresponding Author}

Gwang Ha Kim

ORCID https://orcid.org/0000-0001-9721-5734

E-mail doc0224@pusan.ac.kr
Proton pump inhibitors (PPIs) are used worldwide to treat of acid-related disorders such as peptic ulcer and gastroesophageal reflux disease and to prevent gastroduodenal injuries due to nonsteroidal anti-inflammatory drugs. PPIs are the most potent inhibitors of gastric acid secretion currently available, and they are one of the most commonly prescribed classes of drugs because of their high efficacy and low toxicity. However, long-term PPI use causes histopathological changes such as parietal cell protrusion into the gland lumen, cystic dilation of gastric fundic glands, and foveolar epithelial hyperplasia. These changes can manifest on endoscopic examination as fundic gland polyps, hyperplastic polyps, multiple white and flat elevated lesions, cobblestone-like mucosa, or black spots. Clinicians must be aware of PPI-induced endoscopic features in patients with chronic long-term PPI use. Conversely, identifying patients with long-term PPI use based on their endoscopic findings is important. Recently, potassium-competitive acid blockers (P-CABs), a new class of acid suppressants that inhibit gastric acid secretion more strongly than PPIs, have recently been introduced clinically. Further long-term prospective studies on these gastric mucosal lesions in patients with either PPI or P-CAB use are required to investigate their association with histopathological changes and to establish the clinical significance of these findings. (Gut Liver 2021;15:646-652)

Key Words: Proton pump inhibitor; Stomach; Endoscopy; Long-term adverse effects

\section{INTRODUCTION}

Proton pump inhibitors (PPIs), since their introduction in the late 1980s, have been used worldwide for the treatment of acid-related disorders such as gastroesophageal reflux disease (GERD) and peptic ulcer. They have also been administered prophylactically to prevent gastroduodenal mucosal damage due to nonsteroidal anti-inflammatory drugs (NSAIDs). PPIs are the most potent inhibitors of gastric acid secretion currently available. High efficacy and low toxicity of PPIs, combined with the high prevalence of GERD and NSAID use, has resulted in these becoming one of the most commonly prescribed agents. ${ }^{1}$ GERD is a chronic condition, and the majority of affected patients experience a symptomatic relapse if PPI therapy is discontinued. Therefore, many GERD patients require continuous maintenance PPI therapy. ${ }^{2}$

An important issue with long-term PPI usage is that these drugs raise the level of the peptide hormone gastrin; ${ }^{3}$ as a result of the homoeostatic response of antral G cells, to the reduced acidity of gastric juice. Gastrin exerts trophic effects on the entire gastrointestinal tract tissue, including on both parietal and enterochromaffin-like cells distributed throughout the oxyntic mucosa. ${ }^{4}$ It has been reported that long-term PPI use also induces histopathological changes such as protrusion of parietal cells into the gland lumen and cystic dilation of gastric fundic glands. ${ }^{5-8}$ PPIinduced endoscopic features such as formation of fundic gland polyps, hyperplastic polyps, multiple white and flat elevated lesions, cobblestone-like mucosa, and black spots have been reported in recent studies (Table 1). ${ }^{9-12}$ In the clinical setting, it is important to be aware of PPI-related gastric endoscopic features exhibited by long-term users. Conversely, it is also important to identify long-term PPI users based on their endoscopic findings. In this review of the latest reports on the topic, I have summarized the 
Table 1. Summary of Proton Pump Inhibitor-Related Gastric Mucosal Changes

\begin{tabular}{|c|c|c|c|}
\hline Endoscopic findings & Incidence, \% & Associated histopathological features & Other risk factors \\
\hline Fundic gland polyps & $9-36$ & $\begin{array}{l}\text { Parietal cell protrusion and cystic dilatation of fundic } \\
\text { glands }\end{array}$ & $\begin{array}{l}\text { Absent } H \text {. pylori infection } \\
\text { Absent atrophic gastritis } \\
\text { Female sex }\end{array}$ \\
\hline Hyperplastic polyps & 8.9 & Foveolar epithelial hyperplasia & H. pylori infection \\
\hline $\begin{array}{l}\text { Multiple white and flat elevated } \\
\text { lesions }\end{array}$ & $14.3-26.3$ & Foveolar epithelial hyperplasia & $\begin{array}{l}\text { H. pylori eradication } \\
\text { Atrophic gastritis } \\
\text { Female sex } \\
\text { Aging }\end{array}$ \\
\hline Cobblestone-like mucosa & $9.1-35.1$ & $\begin{array}{l}\text { Parietal cell protrusion and cystic dilatation of fundic } \\
\text { glands }\end{array}$ & $\begin{array}{l}\text { Absent atrophic gastritis } \\
\text { Male sex } \\
\text { Aging } \\
\text { Diabetes mellitus }\end{array}$ \\
\hline Black spots & $0.2-6.2$ & Brownish substances in fundic gland cysts & $\begin{array}{l}\text { H. pylori eradication } \\
\text { A lower body mass index }\end{array}$ \\
\hline
\end{tabular}

H. pylori, Helicobacter pylori.
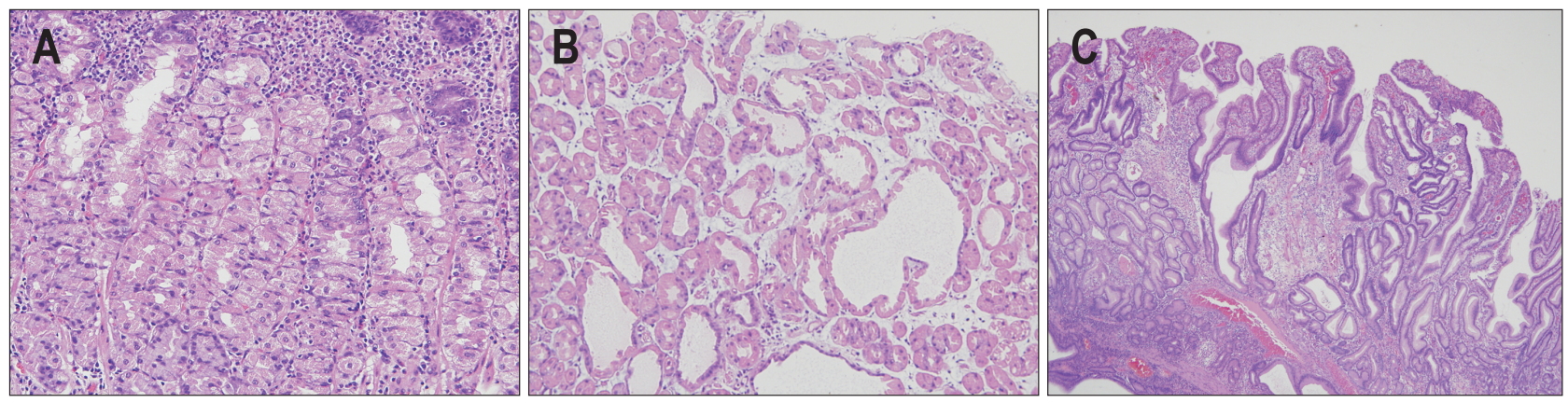

Fig. 1. Histopathological features of proton pump inhibitor-related gastric mucosal changes. (A) Oxyntic mucosa with parietal cell protrusions (H\&E, $\times 200)$. (B) Cystic changes in fundic glands $(H \& E, \times 200)$. (C) Foveolar epithelial hyperplasia $(H \& E, \times 40)$.

histopathological and endoscopic findings in patients with PPI-induced gastric mucosal changes.

\section{PPI-RELATED HISTOPHATOLOGICAL GASTRIC MUCOSAL CHANGES}

Chronic PPI use is associated with changes in the morphology of gastric parietal cells, including an increase in cell size and number and is associated with enterochromaffin-like cell proliferation in the gastric mucosa. ${ }^{13}$ Histopathologically, parietal cell enlargement can easily be identified as a convex bulging and swelling of the apical membrane into the lumen of oxyntic glands. This so-called parietal cell protrusion (PCP) appears as a serrated contour of the internal glands (Fig. 1A), ${ }^{7,13}$ Gastric hydrochloric acid accumulates in parietal cells due to inhibition of its active release from the secretory canaliculi by PPIs, which results in PCP. ${ }^{13}$ Development of this aberrant cellular morphology shows a positive correlation with hypergastrinemia. ${ }^{13} \mathrm{PCP}$ is also observed in other conditions causing hypergastrinemia, such as Helicobacter pylori gastritis, peptic ulcer, or Zollinger-Ellison syndrome. ${ }^{14}$ Therefore, PCP is not a finding unique to acid-suppressive therapy and might be associated with the increase of serum gastrin concentration, to some extent. ${ }^{13}$

PCP further leads to cystic dilation of fundic glands and cytoplasmic vacuolation (Fig. 1B) ${ }^{15}$ Cystic dilation of fundic glands may result from outflow-obstruction. This is attributable to glandular isthmus blockage due to protrusion of parietal cells and plugging by mucus secreted from proliferating foveolar cells, secondary to PPI-induced hypergastrinemia. ${ }^{16,17}$ The resulting cystic dilation has the potential to further enlarge and progress to fundic gland polyp formation. ${ }^{18}$ Previous studies have reported that fundic gland dilatation occurred between 8 months and 60 months after starting PPIs, ${ }^{7,19}$ while a recent meta-analysis concluded that long-term use of PPIs ( $\geq 1$ year) was associated with an increased risk of formation of fundic gland polyps. $^{18}$

Several studies have shown that fundic gland polyps are observed particularly in patients without accompanying H. pylori infection. ${ }^{13,20}$ Enzymatic degradation of gastric mucus by $H$. pylori protease may facilitate glandular out- 
flow, thus protecting gastric glands against acid-retention and cystic dilation. ${ }^{21}$ While $\beta$-catenin gene mutations were found in $64 \%$ to $91 \%$ of patients with sporadic fundic gland polyps, these mutations were not found in patients developing these polyps following PPI treatment. ${ }^{22}$ In a histological analysis, PCP was found in $9.2 \%$ and $64.4 \%$ of fundic gland polyps in patients not taking PPIs and in those taking the drugs, respectively. The maximum diameter of fundic gland cysts within the fundic gland polyps was larger in patients on PPI treatment than in those not receiving the drugs. ${ }^{22} \mathrm{~A}$ retrospective study revealed three types of PPI-associated gastric polyps in chronic users, (1) fundic gland type, (2) hyperplastic type, and (3) inflammatory type. ${ }^{23}$ However, whether these polyps differ from those occurring sporadically in the general population, is still unclear.

PPI-induced hypergastrinemia possibly also causes foveolar epithelial hyperplasia (Fig. 1C), which generally presents as hyperplastic polyps. ${ }^{24}$ This may also present as multiple white and flat elevated lesions. ${ }^{12,25}$ Therefore, hyperplastic polyps and multiple white and flat elevated lesions are considered as endoscopic features of foveolar epithelial hyperplasia, caused by chronic PPI consumption.
ENDOSCOPIC FEATURES OF PPI-RELATED GASTRIC MUCOSAL CHANGES

\section{Fundic gland polyps}

Fundic gland polyps are usually found in the gastric corpus and fundus, and they are generally multiple, small $(<1 \mathrm{~cm})$, sessile, and whitish-pink in color, with a smooth, partially translucent surface (Fig. 2A). In 1992, Graham ${ }^{26}$ first reported that fundic gland polyps arose during omeprazole treatment. In a prospective study of 191 longterm PPI users, fundic gland polyps were identified in $13.6 \%$ of patients, with the incidence in $H$. pylori-positive patients being significantly lower than in $H$. pylori-negative patients (hazard ratio, 0.29 ; 95\% confidence interval [CI], 0.11 to 0.76 ); the majority of fundic gland polyps arose within 2 years of initiating treatment with PPIs. ${ }^{24}$ Other studies on long-term PPI therapy have reported a $9 \%$ to $36 \%$ incidence of new, fundic gland polyp formation. ${ }^{9,27}$ A recent meta-analysis of 12 studies also revealed PPI use to be significantly associated with an increased prevalence of fundic gland polyps (odds ratio [OR], 2.4; 95\% CI, 1.42 to 4.27$){ }^{28}$ This association was observed to positively correlate with a longer time-interval of PPI exposure (for $\geq 6$ months: OR, 4.71; $95 \%$ CI, 2.22 to 9.99 and for 12 months: OR, 5.32; 95\% CI, 2.58 to 10.99$).{ }^{28}$ In addition to the appearance of new fundic gland polyps, the size of existing
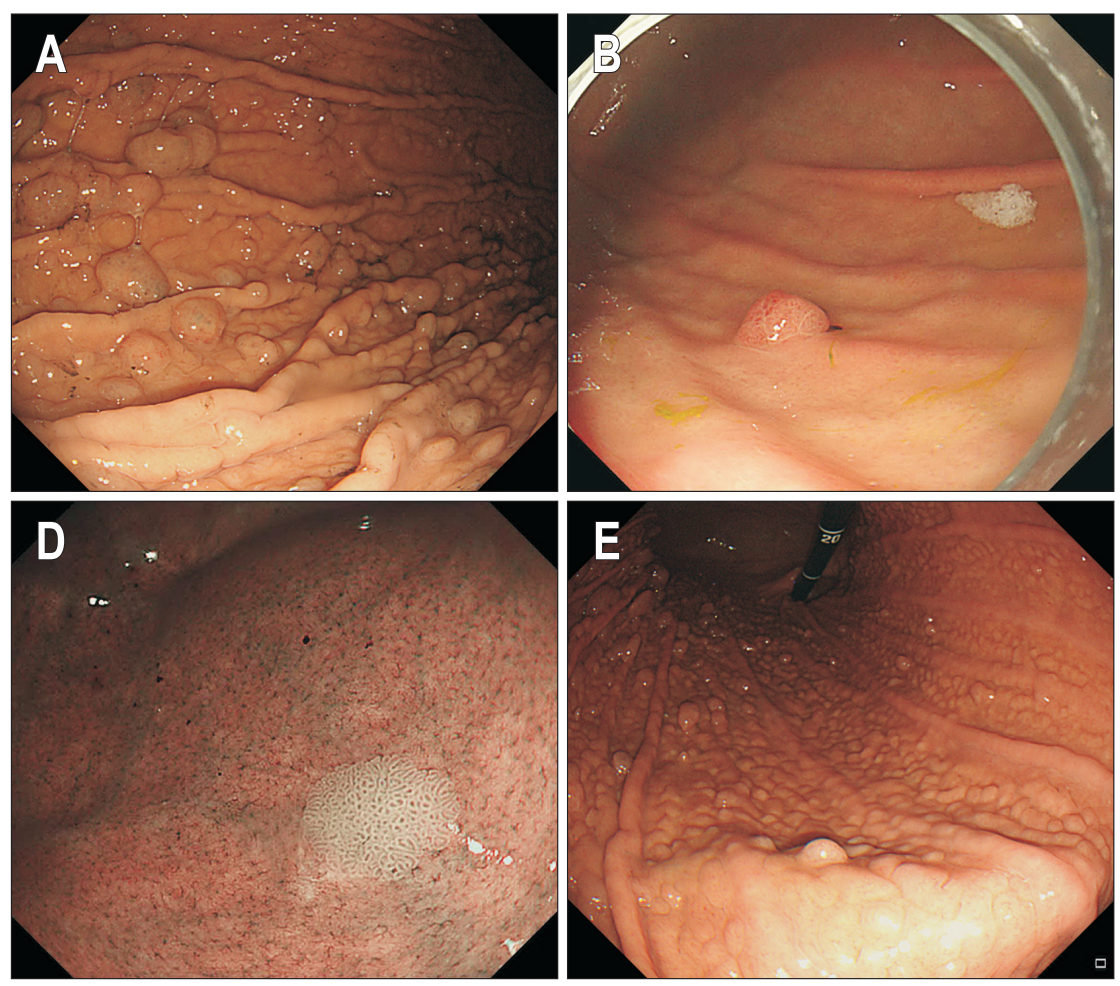
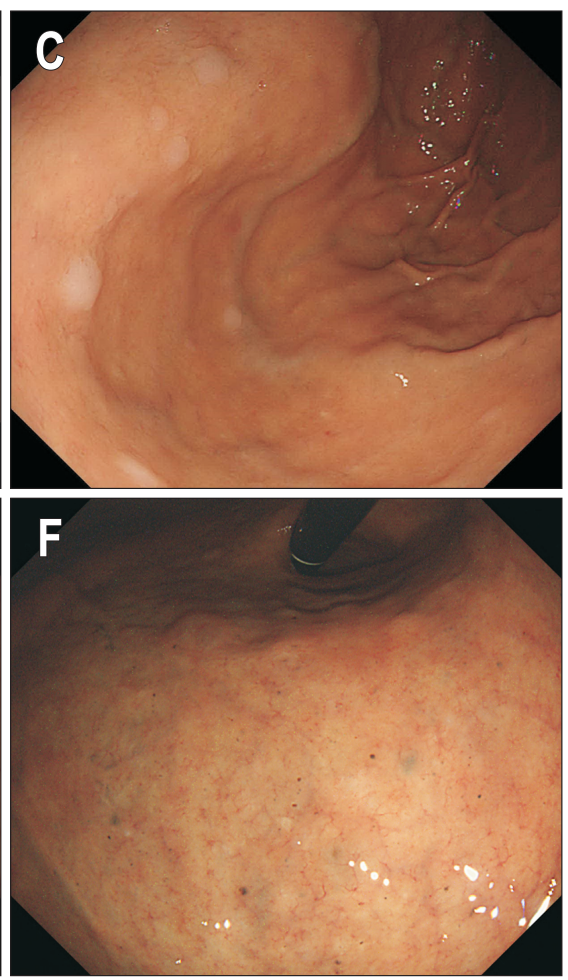

Fig. 2. Endoscopic features of proton pump inhibitor-related gastric mucosal changes. (A) Fundic gland polyps. (B) Hyperplastic polyp. (C, D) Multiple white and flat elevated lesions. (E) Cobblestone-like mucosa. (F) Black spots. 
polyps was often found to increase with PPI intake. Conversely, the fundic gland polyps were also observed to decrease in size or regress, following discontinuation of PPIs. There is no need to resect fundic gland polyps, as they have virtually no malignant potential in the general population. ${ }^{29,30}$ However, a case-series report has documented a very rare, fundic gland type gastric adenocarcinoma. ${ }^{31} \mathrm{~A}$ recent cross-sectional study showed that the occurrence of fundic gland polyps correlated with female sex (OR, 1.77; 95\% CI, 1.31 to 2.39 ) and atrophic gastritis (OR, 0.12; 95\% CI, 0.08 to 0.18$).^{12}$

\section{Hyperplastic polyps}

Cases of patients with newly formed hyperplastic polyps due to long-term PPI use have been reported, especially in conditions associated with hypergastrinemia and concurrent H. pylori infection (Fig. 2B). ${ }^{11,23,24}$ In a prospective study, hyperplastic polyps were identified in $8.9 \%$ of longterm PPI users. Development of new hyperplastic polyps was observed in $6 \%$ and $22 \%$ of patients with existing fundic gland polyps and hyperplastic polyps, respectively, over a 2-year period of PPI usage. ${ }^{24}$ Furthermore, the incidence of hyperplastic polyps tended to be higher in $H$. pyloripositive patients than in $H$. pylori-negative patients. ${ }^{24}$

Although the exact pathophysiological mechanism of hyperplastic polyp-formation is unclear, the gastrin receptor was reportedly expressed on the foveolar epithelium of hyperplastic polyps. ${ }^{32}$ Gastrin induces proliferative effects on the gastric mucosa, which in turn enhances the effects of growth factors such as epidermal growth factor and tumor growth factor- $\alpha$ families, thereby promoting proliferation of crypt epithelial cells. ${ }^{21}$ Therefore, PPI-induced hypergastrinemia is considered to cause hyperplasia of the gastric foveolar epithelium, which leads to the formation of hyperplastic polyps.

\section{Multiple white and flat elevated lesions}

Multiple white and flat elevated lesions are defined as apparently circumscribed and sharply demarcated white-colored, round, and slightly elevated mucosa with a smooth surface (Fig. 2C). ${ }^{12,25}$ These lesions are usually found in the upper gastric corpus and fundus, and close observation allows identification of tubular structures on their surface (Fig. 2D). Dilated vessels usually observed in fundic gland polyps, are absent. ${ }^{25}$ The rate of detection of these characteristic lesions can be increased by endoscopic examination or by using image-enhanced endoscopy such as narrow-band imaging. These lesions have been reported in $14.3 \%$ to $26.3 \%$ of PPI users. ${ }^{10,33}$ In observational studies, multiple white and flat elevated lesions were found to be significantly associated with PPI use (OR, 3.58; 95\%
CI, 1.94 to 6.61), H. pylori eradication therapy (OR, 2.11; 95\% CI, 1.08 to 4.11), female sex (OR, 1.92; 95\% CI, 1.19 to 3.12 ), aging (OR, 1.05; 95\% CI, 1.02 to 1.08 ), and moderate to severe atrophic gastritis (OR, 2.63; 95\% CI, 1.52 to 5.63). ${ }^{12,33-35}$ These lesions are also related with administration of histamine receptor antagonists and $H$. pylori eradication, and are therefore not uniquely associated with chronic PPI use. Furthermore, these lesions can be misdiagnosed as intestinal metaplasia. Points of differentiation are as follows: (1) multiple white and flat elevated lesions appear in the upper corpus and fundus (intestinal metaplasia in the antrum often appears as whitish elevated patches, while intestinal metaplasia in the corpus is usually flat), and (2) multiple white and flat elevated lesions have a ridged or papillary surface structure, lacking the light-blue crest sign, on magnifying endoscopy with narrow-band imaging. ${ }^{36}$

\section{Cobblestone-like mucosa}

Cobblestone-like mucosa is defined as numerous, approximately 3 to $5 \mathrm{~mm}$-sized, uneven, elevated, mucosal lesions in the gastric corpus (Fig. 2E). ${ }^{12,25}$ Cobblestone-like mucosa has coloration similar to that of the surrounding mucosa, is usually observed in-between gastric folds, and has a fluffy appearance. The magnifying endoscopy with narrow-band imaging shows dilation of the oval crypt opening and the intervening part. ${ }^{11}$ Several observational, case-control studies have shown that this cobblestone-like mucosa is significantly associated with PPI intake with a frequency of $9.1 \%$ to $35.1 \%$ in chronic users. ${ }^{10,37,38}$ While chronic PPI users with cobblestone-like mucosa did not differ from those without cobblestone-like mucosa with respect to factors including age, sex, period of use, and $H$. pylori eradication, the characteristic mucosal appearance was observed more frequently in patients without atrophic gastritis. ${ }^{38}$ In a cross-sectional study, multivariate analyses showed that the cobblestone-like mucosa was associated with PPI use (OR, 4.57; 95\% CI, 2.34 to 9.96) and diabetes mellitus (OR, 3.41; 95\% CI, 1.47 to 7.91 ). ${ }^{12}$ A prospective, multicenter study also showed that the cobblestone-like mucosa was associated with PPI use (OR, 13.72; 95\%, CI, 5.49 to 35.28 ), sex (female: OR, $0.32 ; 95 \% \mathrm{CI}, 0.09$ to 0.89 ), and age of the patients ( $\geq 50$ years: OR, $4.46,95 \%$ CI, 1.15 to 29.49$).^{10}$

Cobblestone-like mucosa has been histopathologically characterized by PCP and cystic dilatation of fundic glands, and these changes are especially prominent in nonatrophic gastric regions with abundant fundic glands. ${ }^{39}$ When cobblestone-like mucosa is observed in regions with atrophic gastritis, the remaining gastric mucosal area with conserved fundic glands shows a characteristic, reddish- 
Histopathological changes

Parietal cell protrusion

Cystic dilation of fundic glands

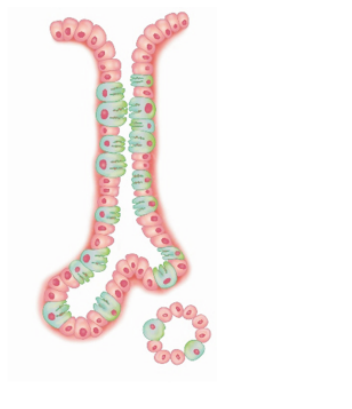

Foveolar epithelial hyperplasia

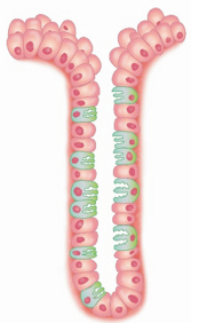

\section{Endoscopic findings}

\author{
- Fundic gland polyps \\ - Cobblestone-like mucosa \\ - Black spots
}

Fig. 3. Suggested mechanisms of proton pump inhibitor (PPI)-related gastric mucosal changes.

colored, elevated morphology, against a background of discolored, atrophic mucosa.

\section{Black spots}

Black spots are defined as black-colored pigmentation in gastric mucosa on conventional endoscopy, and are localized to the gastric body and fundus, which contain fundic glands. ${ }^{40}$ Superficially, they look like blood clots, but the overlying mucosa is flat in appearance (Fig. 2F). They are usually multiple (approximately in 65\% of patients), ${ }^{40}$ and are sometimes observed inside fundic gland polyps (Fig. 2A). Nonetheless, it is difficult to detect black spots on routine endoscopy without detailed examination. Observation studies have reported a frequency of black spots to be $0.2 \%$ to $6.2 \%$. ${ }^{10,12,40}$ On multivariate analyses, black spots were associated with PPI use (OR, 2.94; 95\% $\mathrm{CI}, 1.66$ to 5.21 ), $H$. pylori eradication therapy (OR, 3.01; 95\% CI, 1.73 to 5.24 ), and a lower body mass index (OR, 0.89 per $\mathrm{kg} / \mathrm{m}^{2}$; $95 \% \mathrm{CI}, 0.82$ to 0.96$) .{ }^{12}$ The frequency of black spots was reported as $14 \%$ in the $H$. pylori eradication group or in PPI users, while it was $<5 \%$ each in the group without $H$. pylori eradication and in PPI nonusers. ${ }^{12}$ Histopathologically, black spots appear to comprise brownish substances and/or eosinophilic exudates within fundic gland cysts, which are thought to develop due to the accumulation of secretions from the lining cells of fundic gland cysts. ${ }^{12}$ However, the composition of the contents of black spots and their mechanism of formation have yet to be elucidated.

\section{CONCLUSIONS}

In long-term PPI users, characteristic mucosal changes, such as fundic gland polyps, hyperplastic polyps, multiple white and flat elevated lesions, cobblestone-like mucosa, and black spots, can be observed on endoscopy. Histopathologically, while the development of fundic gland polyps, cobblestone-like mucosa, and black spots are considered to be caused by PCP and the cystic dilatation of fundic glands, the formation of hyperplastic polyps and multiple white and flat elevated lesions are attributed to foveolar epithelial hyperplasia (Fig. 3). With an ever-increasing number of PPI users, endoscopic identification of these mucosal changes is important. Recently, potassium competitive acid blockers (P-CABs), a new class of acid suppressants, which inhibit gastric $\mathrm{H}^{+}, \mathrm{K}^{+}$-ATPase activity via reversible and $\mathrm{K}^{+}$-competitive ionic binding to the enzyme and have stronger inhibitory effect on gastric acid secretion than PPIs, has recently been introduced in the clinical setting. 
Although an association between the aforementioned histopathological changes and P-CAB use has not yet been established, there is a possibility that long-term $\mathrm{P}-\mathrm{CAB}$ users may also demonstrate similar gastric mucosal changes as observed in long-term PPI users. Further long-term, prospective studies examining these gastric mucosal lesions in both PPI and P-CAB users are required to investigate their association with histopathological changes and to establish the clinical significance of these changes.

\section{CONFLICTS OF INTEREST}

G.H.K. is an editorial board member of the journal but was not involved in the peer reviewer selection, evaluation, or decision process of this article. No other potential conflicts of interest relevant to this article were reported.

\section{ACKNOWLEDGEMENTS}

The author would like to thank Dr. Sojeong Lee, MD, PhD (Pusan National University Hospital, Busan, Korea), for her assistance in providing histopathological figures.

\section{ORCID}

Gwang Ha Kim https://orcid.org/0000-0001-9721-5734

\section{REFERENCES}

1. Heidelbaugh JJ, Kim AH, Chang R, Walker PC. Overutilization of proton-pump inhibitors: what the clinician needs to know. Therap Adv Gastroenterol 2012;5:219-232.

2. Nocon M, Labenz J, Jaspersen D, et al. Long-term treatment of patients with gastro-oesophageal reflux disease in routine care: results from the ProGERD study. Aliment Pharmacol Ther 2007;25:715-722.

3. Pounder R, Smith J. Drug-induced changes of plasma gastrin concentration. Gastroenterol Clin North Am 1990;19:141153.

4. Wang TC, Koh TJ, Varro A, et al. Processing and proliferative effects of human progastrin in transgenic mice. J Clin Invest 1996;98:1918-1929.

5. Graham DY, Genta RM. Long-term proton pump inhibitor use and gastrointestinal cancer. Curr Gastroenterol Rep 2008; 10:543-547.

6. Kamada T, Haruma K, Inoue K, Shiotani A. Helicobacter pylori infection and endoscopic gastritis: Kyoto classification of gastritis. Nihon Shokakibyo Gakkai Zasshi 2015;112:982993.

7. Stolte M, Bethke B, Rühl G, Ritter M. Omeprazole-induced pseudohypertrophy of gastric parietal cells. Z Gastroenterol 1992;30:134-138.

8. Declich P, Ambrosiani L, Bellone S, et al. Parietal cell hyperplasia with deep cystic dilations: a lesion closely mimicking fundic gland polyps. Am J Gastroenterol 2000;95:566-568.

9. Jalving M, Koornstra JJ, Wesseling J, Boezen HM, DE Jong S, Kleibeuker JH. Increased risk of fundic gland polyps during long-term proton pump inhibitor therapy. Aliment Pharmacol Ther 2006;24:1341-1348.

10. Kiso M, Ito M, Boda T, et al. Endoscopic findings of the gastric mucosa during long-term use of proton pump inhibitor: a multicenter study. Scand J Gastroenterol 2017;52:828-832.

11. Miyamoto S, Kato M, Matsuda K, et al. Gastric hyperplastic polyps associated with proton pump inhibitor use in a case without a history of Helicobacter pylori infection. Intern Med 2017;56:1825-1829.

12. Hatano Y, Haruma K, Kamada T, et al. Factors associated with gastric black spot, white flat elevated mucosa, and cobblestone-like mucosa: a cross-sectional study. Digestion 2018;98:185-193.

13. Cats A, Schenk BE, Bloemena E, et al. Parietal cell protrusions and fundic gland cysts during omeprazole maintenance treatment. Hum Pathol 2000;31:684-690.

14. Krishnamurthy S, Dayal Y. Parietal cell protrusions in gastric ulcer disease. Hum Pathol 1997;28:1126-1130.

15. Ang ST, Lieberman DA, Ippoliti AF, et al. Long-term omeprazole therapy in patients with Barrett's esophagus is associated with parietal cell hyperplasia. Gastroenterology 1994;106:A1016.

16. Shiotani A, Katsumata R, Gouda K, et al. Hypergastrinemia in long-term use of proton pump inhibitors. Digestion 2018; 97:154-162.

17. Synnerstad I, Holm L. Omeprazole induces high intraglandular pressure in the rat gastric mucosa. Gastroenterology 1997;112:1221-1230.

18. Tran-Duy A, Spaetgens B, Hoes AW, de Wit NJ, Stehouwer $\mathrm{CD}$. Use of proton pump inhibitors and risks of fundic gland polyps and gastric cancer: systematic review and metaanalysis. Clin Gastroenterol Hepatol 2016;14:1706-1719.

19. Stolte M, Bethke B, Seifert E, et al. Observation of gastric glandular cysts in the corpus mucosa of the stomach under omeprazole treatment. Z Gastroenterol 1995;33:146-149.

20. el-Zimaity HM, Jackson FW, Graham DY. Fundic gland polyps developing during omeprazole therapy. Am J Gastroenterol 1997;92:1858-1860.

21. Dockray GJ. Topical review: gastrin and gastric epithelial physiology. J Physiol 1999;518(Pt 2):315-324.

22. Fukuda M, Ishigaki $H$, Sugimoto $M$, et al. Histological analy- 
sis of fundic gland polyps secondary to PPI therapy. Histopathology 2019;75:537-545.

23. Choudhry U, Boyce HW Jr, Coppola D. Proton pump inhibitor-associated gastric polyps: a retrospective analysis of their frequency, and endoscopic, histologic, and ultrastructural characteristics. Am J Clin Pathol 1998;110:615-621.

24. Hongo M, Fujimoto K; Gastric Polyps Study Group. Incidence and risk factor of fundic gland polyp and hyperplastic polyp in long-term proton pump inhibitor therapy: a prospective study in Japan. J Gastroenterol 2010;45:618-624.

25. Haruma K, Kato M, Inoue K, Murakami M, Kamada T. Kyoto classification of gastritis. 2nd ed. Tokyo: Nihon Medical Center, 2019.

26. Graham JR. Gastric polyposis: onset during long-term therapy with omeprazole. Med J Aust 1992;157:287-288.

27. Mogadam M, Houk RR. Long-term use of omeprazole is associated with development of benign epithelial gastric polyps. Am J Gastroenterol 1996;91:A151.

28. Martin FC, Chenevix-Trench G, Yeomans ND. Systematic review with meta-analysis: fundic gland polyps and proton pump inhibitors. Aliment Pharmacol Ther 2016;44:915-925.

29. Kato K, Iwasaki Y, Taniguchi M, et al. Successful treatment of proton pump inhibitor induced sporadic fundic gland polyps with an argon plasma coagulator in a patient with polycythaemia vera. Int J Surg Case Rep 2017;33:75-78.

30. Park YJ, Kim GH. Proton pump inhibitor-induced fundic gland polyposis. Korean J Intern Med 2017;32:197-198.

31. Nakagawa M, Abe M, Takada S, et al. Endoscopic features of gastric adenocarcinoma of fundic gland type. Stomach Intestine 2015;50:1521-1531.
32. Takamura A, Ito M, Boda T, et al. High expression of gastrin receptor protein in injured mucosa of Helicobacter pyloripositive gastritis. Dig Dis Sci 2013;58:634-640.

33. Majima K, Muraki Y, Shimamoto T. Multiple white and flat elevated lesions observed in the stomach: a prospective study of clinical characteristics and risk factors. Intern Med 2018; 57:2613-2619.

34. Hasegawa R, Yao K, Ihara S, et al. Magnified endoscopic findings of multiple white flat lesions: a new subtype of gastric hyperplastic polyps in the stomach. Clin Endosc 2018; 51:558-562.

35. Adachi K, Mishiro T, Okada M, Kinoshita Y. Prevalence of multiple white and flat elevated lesions in individuals undergoing a medical checkup. Intern Med 2018;57:1213-1218.

36. Uedo N, Yamaoka R, Yao K. Multiple white flat lesions in the gastric corpus are not intestinal metaplasia. Endoscopy 2017; 49:615-616.

37. Miyamoto S, Kato M, Tsuda M, et al. Gastric mucosal cracked and cobblestone-like changes resulting from proton pump inhibitor use. Dig Endosc 2017;29:307-313.

38. Takahari K, Haruma K, Ohtani H, et al. Proton pump inhibitor induction of gastric cobblestone-like lesions in the stomach. Intern Med 2017;56:2699-2703.

39. Miyamoto S, Kudo T, Kato M, et al. Endoscopic ultrasonography features of gastric mucosal cobblestone-like changes from a proton-pump inhibitor. Clin J Gastroenterol 2017;10: 220-223.

40. Hatano Y, Haruma K, Ayaki M, et al. Black spot, a novel gastric finding potentially induced by proton pump inhibitors. Intern Med 2016;55:3079-3084. 